\title{
RINITE ALÉRGICA E/OU ASMA EM ADOLESCENTES DO ENSINO MÉDIO REGULAR DA REDE PÚBLICA
}

Allergic rhinitis and/or asthma in teenagers attending regular government-run high schools

Rinitis alérgica y/o asma en adolescentes de la enseñanza secundaria en el sistema público

Laíze Almeida Santos ${ }^{1}$, José Marcos de Jesus Santos², Flávia Márcia Oliveira ${ }^{3 *}$

1 Departamento de Farmácia, Campus Lagarto, Universidade Federal de Sergipe (UFS)

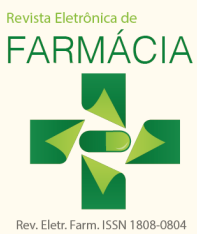

2 Departamento de Enfermagem, Campus Lagarto, Universidade Federal de Sergipe (UFS)

${ }^{3}$ Departamento de Educação em Saúde, Campus Lagarto, Universidade Federal de Sergipe (UFS)

*E-mail: fmo.ufs@hotmail.com

\section{RESUMO}

Introdução: Rinite alérgica e asma são doenças inflamatórias crônicas que representam um grande impacto tanto na saúde individual quanto no nível econômico e social.

Objetivo: O estudo teve como objetivo analisar a rinite alérgica e/ou asma em estudantes do ensino médio regular da rede pública em Simão Dias, Sergipe, Brasil.

Métodos: Trata-se de uma pesquisa transversal descritiva realizada em fevereiro e março de 2017 . Selecionaram-se 46 estudantes para os quais foram aplicados um questionário referente às variáveis sociodemográficas, manifestações clínicas e impactos sobre o cotidiano; e um formulário sobre o uso de medicamentos.

Resultados: A rinite alérgica apresentou maior prevalência ( $61 \% ; n=28$ ) e identificou-se coexistência com asma em $11 \%$ ( $n=5)$. Grande parte foi classificada nas faixas de baixa frequência de sinais e sintomas $(45,7 \%$; $n=21)$ e de baixo impacto sobre as atividades diárias (47,8\%; $n=22)$. O sexo feminino apresentou maior frequência sintomatológica $(35,5 \% ; n=11)$. Para a rinite alérgica, a maioria das terapias medicamentosas encontrava-se adequada. Por outro lado, no caso da asma, uma proporção desconhecia ou não fazia o uso de medicamentos para manutenção, mesmo apresentando uma frequência importante de sinais e sintomas e crises.

Conclusão: Destaca-se a importância da sensibilização dos adolescentes quanto ao autocuidado visando o controle das doenças e melhoria da adesão ao tratamento.

Palavras-chave: Doenças respiratórias. Impactos na saúde. Adolescente.

\section{ABSTRACT}

Introduction: Allergic rhinitis and asthma are chronic inflammatory diseases that seriously affect not only the health of individuals but also the economic and social systems.

Objective: This study aimed to analyze the prevalence of allergic rhinitis and asthma in students attending government-run high schools of the municipality of Simão Dias, Sergipe, Brazil.

Methods: This was a descriptive cross-sectional study conducted in February and March 2017. A total of 46 students were selected and completed a questionnaire assessing sociodemographic variables, clinical manifestations, and impact on their everyday life, in addition to a form about the use of medication.

Results: Allergic rhinitis had the highest prevalence $(61 \% ; n=28)$, coexisting with asthma at $11 \%(n=5)$. Most cases were categorized as having a low frequency of signs and symptoms $(45.7 \% ; n=21)$ and a low impact on the activities of daily living (47.8\%; $n=22)$. Female students presented a higher frequency of symptoms $(35.5 \% ; n=11)$. For allergic rhinitis, most 
drug therapies used were considered to be appropriate. On the other hand, a proportion of students with asthma was either unaware of the existence of maintenance medication or did not use any, even though they had a considerable frequency of signs, symptoms, and asthma attacks.

Conclusions: It is important to raise self-care awareness among teenagers, with the aim to control these diseases and improve treatment adherence.

Keywords: Respiratory tract diseases. Impacts on health. Adolescent.

\section{RESUMEN}

Introdución: La rinitis alérgica y el asma son enfermedades inflamatorias crónicas que representan un gran impacto, tanto para la salud individual, como en el plano económico y social.

Objetivo: El estudio tuvo el objetivo de analizar la rinitis alérgica/asma en los estudiantes de enseñanza secundaria de escuelas públicas de Simão Dias, Sergipe, Brasil.

Métodos: Se trata de una investigación descriptiva y transversal, realizada durante los meses de febrero y marzo de 2017. Se seleccionaron 46 estudiantes a los que seles aplico un cuestionario sobre las variables sociodemográficas, síntomas clínicos e impactos en la vida diaria; y un formulario sobre el uso de medicamentos.

Resultados: La rinitis alérgica presentó una prevalencia mayor $(61 \% ; n=28)$ y se identificó una coexistencia con el asma en un $11 \%(n=5)$. Grande parte se clasificó en las fajas de baja frecuencia de indicios y síntomas $(45,7 \%, n=21)$ y de bajo impacto en las actividades diarias $(47,8 \%, n=22)$. El sexo femenino presentó una mayor frecuencia sintomatológica $(35,5 \%, n=11)$. Para la la rinitis alérgica, la mayoría de las terapias médicas eran adecuadas. Por otra parte, en el caso del asma, una parte desconocía o no hacía uso de medicamentos para tratarlo, aunque presentase una frecuencia importante de indicios, síntomas y crisis.

Conclusión: Se destaca la importancia de sensibilizar a los adolescentes sobre el autocuidado para controlar las enfermedades y mejorar la su incorporación al tratamiento.

Palabras-clave: Enfermedades respiratorias, Impactos en la salud, Adolescente.

\section{INTRODUÇÃO}

Rinite alérgica é definida como um transtorno crônico sintomático do nariz provocado, inicialmente, pela exposição a alérgenos e consequente inflamação da mucosa nasal mediada por resposta dependente de Imunoglobulina E (IgE) $)^{(1)}$. A rinite alérgica está entre as dez razões mais frequentes de atendimento na Atenção Primária à Saúde. Afeta a qualidade de vida das pessoas e interfere no período produtivo, o que pode causar prejuízos pelo absenteísmo ao trabalho e à escola(2).

$\mathrm{Na}$ asma, a maioria dos casos inicia-se com uma resposta mediada por IgE. Posteriormente, surgem alterações características de um processo inflamatório crônico ${ }^{(3)}$. Cabe ressaltar que em 2011 foram registradas 160 mil hospitalizações, o que levou a asma para a quarta colocação das causas de internações no SUS. ${ }^{(4)}$

Além disso, as duas doenças exercem um grande impacto econômico. Nos Estados Unidos há uma estimativa que o valor gasto anualmente com a rinite alérgica ultrapasse U\$ 3 bilhões. No caso da asma, o custo anual por paciente com asma pode variar entre U\$326,00 na Austrália e U\$1.315,00 na Suécia(5). No Brasil, em 2007, a asma gerou um custo aproximado de $R \$$ 98,6 milhões para o Sistema Único de Saúde.(2)

Todas estas ações são consequência da inexistência de cura que, dessa forma, exige um acompanhamento contínuo dos pacientes. No entanto, observa-se a falta de controle da doença na maioria das pessoas devido, especialmente, a não utilização 
adequada dos medicamentos profiláticos, ao desconhecimento dos aspectos fundamentais da doença e à subestimação por parte dos pacientes, familiares e, inclusive, de alguns profissionais de saúde..(6,7)

Considerando a importância do cuidado farmacêutico na saúde individual e pública, o estudo tem como objetivo principal analisar o perfil do uso de medicamentos, sinais, sintomas e impacto sobre a qualidade de vida da rinite alérgica e/ou asma em estudantes matriculados no ensino médio regular da rede pública do município de Simão Dias, Sergipe.

\section{METODOLOGIA}

Trata-se de um estudo transversal descritivo realizado no período de fevereiro a março de 2017.

A população abrangeu os estudantes matriculados no ensino médio regular da rede pública em Simão Dias, Sergipe. Segundo informações da Secretaria de Estado da Educação de Sergipe, no ano letivo de 2015 foram registradas 1.048 matrículas no único colégio da região. Considerando a prevalência média de $21 \%$ para rinite alérgica e/ou asma, 95\% de nível de confiança e $5 \%$ de erro amostral, foram sorteados 236 participantes. Dentre estes, identificou-se que $22,5 \%$ ( $n=53)$ apresentavam sinais e sintomas para rinite alérgica e/ou asma autorreferidos. Os critérios de inclusão compreenderam uso de medicamentos para estas doenças, o que correspondeu a uma amostra final de 46 estudantes (prevalência mínima de $70 \%$ para o uso de medicamentos para estas doenças, $95 \%$ de nível de confiança e $5 \%$ de erro amostral). Todos os componentes da amostra aceitaram participar da pesquisa e apresentaram o Termo de Consentimento Livre Esclarecido assinado por eles e/ou pelos responsáveis.

Utilizaram-se dois instrumentos para a realização da pesquisa. O levantamento dos dados sociodemográficos principais (idade, sexo, moradia, escolaridade dos pais), da frequência dos sinais e sintomas e dos impactos sobre as atividades diárias foram realizados por meio de um questionário composto por questões fechadas e adaptado de instrumentos já validados como International Study of Asthma and Allergies in Childhood (ISAAC), Controle da Asma e Rinite Alérgica (CARAT) e Questionário do Hospital Saint George na Doença Respiratória (SGRQ). Para a determinação do perfil do uso de medicamentos para rinite alérgica e/ou asma, sem considerar o contexto de prescrição médica e/ou automedicação, um formulário foi aplicado por meio de entrevista devido à maior complexidade das questões. Também foi construída uma prancha com fotos dos principais medicamentos utilizados para estas doenças a fim de verificar a capacidade de reconhecimento.

Estabeleceram-se índices para aferir a intensidade da frequência de sinais e sintomas para rinite alérgica e/ou asma e o impacto destas doenças sobre as atividades diárias por meio da atribuição dos seguintes valores para resposta de cada variável estudada: frequentemente $=2$ pontos; às vezes $=1$ ponto; nunca $=0$ (valor total: 20 pontos). Posteriormente, foram determinadas faixas de valores a partir de percentis iguais para os casos verificados: frequência dos sinais e sintomas - 0 a 9 (frequência baixa), 10 a 13 (frequência média) e 14 a 20 (frequência alta); e impacto dos sinais e sintomas - 0 a 6 (impacto baixo), 7 a 9 (impacto médio) e 10 a 15 (impacto alto).

Para a análise dos dados utilizou-se o programa SPSS $®$ versão 20 para determinação da frequência absoluta/ relativa, média/desvio padrão, mínimo e máximo. Foram também empregados os testes do Qui-quadrado, comparação de proporções (z), Kendall'sTau-b e Exato de Fischer. Considerou-se significância estatística quando o valor de p foi menor que 0,05 .

A pesquisa foi autorizada pela Diretoria Regional de Educação 2 (DRE2) de Sergipe e executada em conformidade com a Resolução № 446/12 do Conselho Nacional de Saúde. Obteve aprovação pelo Comitê de Ética em Pesquisa № 1.935.218 em 21 de fevereiro de 2017. 


\section{RESULTADOS}

Foi verificada que a taxa de utilização de medicamentos para rinite alérgica e/ou asma foi de $87 \%(n=46)$ dentre aqueles estudantes que apresentam sinais e sintomas frequentes de rinite alérgica e/ou asma autorreferidos ( $n=53)$. Observou-se que a idade média foi igual a 16,4 1,2, sendo a mínima 15 e a máxima 19 anos (adolescentes jovens). A maior parte da amostra $(67,4 \% ; n=31)$ correspondeu ao sexo feminino. Quanto ao nível de escolaridade dos pais, o ensino fundamental foi o mais frequente $(47,8 \% ; n=22)$. Por outro lado, as mães apresentaram distribuições iguais para os ensinos fundamental e médio (37\%; $n=17)$.

A rinite alérgica apresentou maior prevalência, $61 \%(n=28)$, dentre os estudantes que usavam medicamentos e, em $11 \%$ dos casos $(n=5)$, havia coexistência com asma. Em relação à asma, 39\% dos estudantes relataram possuir a doença ( $n=23)$.

Ao investigar a manifestação dos sinais e sintomas de rinite alérgica e/ou asma (Tabela 1), observou-se que os mais presentes foram espirros e/ou prurido nasal, ocular e na faringe/laringe, uma vez que $37 \%$ dos estudantes afirmaram frequentemente manifestá-los $(n=17)$. A poeira e/ou mofo foram apontados como agentes causais frequentes pela maioria dos entrevistados $(58,7 \% ; n=27)$. Grande parte foi classificada na faixa de baixa frequência de manifestações clínicas $(45,7 \%$; $n=21)$. No entanto, uma parcela importante da amostra relatou que apresenta reações com frequência alta $(26,1 \%$; $n=12)$. Não houve diferença significativa e associação entre os tipos de doenças (rinite alérgica, asma e rinite/asma) e a faixa de sinais e sintomas ( $p=0,979$; $r=0,019)$.

Tabela 1. Frequências absoluta e relativa relacionadas à manifestação de sinais e sintomas em estudantes do ensino médio regular que utilizam medicamentos para rinite alérgica e/ou asma ( $n=46)$, Simão Dias, SE, Brasil, 2017

\begin{tabular}{|c|c|c|c|}
\hline \multirow{2}{*}{ Variáveis } & \multicolumn{3}{|c|}{$\begin{array}{l}\text { Frequência da manifestação dos sinais e sintomas } \\
\text { de rinite alérgica e/ou asma }\end{array}$} \\
\hline & $\begin{array}{l}\text { Nunca } \\
\text { n (\%) }\end{array}$ & $\begin{array}{l}\text { Às vezes } \\
\text { n }(\%)\end{array}$ & $\begin{array}{l}\text { Frequentemente } \\
\text { n (\%) }\end{array}$ \\
\hline Espirros e/ou prurido & $1(2,2)$ & $28(60,9)$ & $17(37)$ \\
\hline Coriza e/ou congestão nasal & $1(2,2)$ & $33(71,7)$ & $12(26,1)$ \\
\hline Resfriado no inverno & $5(10,9)$ & $29(63)$ & $12(26,1)$ \\
\hline Poeira e/ou mofo como causa & $1(2,2)$ & $18(39,1)$ & $27(58,7)$ \\
\hline Produto de limpeza como causa & $9(19,6)$ & $26(56,5)$ & $11(23,9)$ \\
\hline Chiado no peito & $16(34,8)$ & $22(47,8)$ & $8(17,4)$ \\
\hline Tosse, dispneia ou opressão torácica & $5(10,9)$ & $31(67,4)$ & $10(21,7)$ \\
\hline Necessidade da técnica de inalação & $15(32,6)$ & $28(60,9)$ & $3(6,5)$ \\
\hline Esforço físico como causa & $18(39,1)$ & $22(47,8)$ & $6(13)$ \\
\hline \multirow[t]{3}{*}{ Inverno como causa } & $16(34,8)$ & $20(43,5)$ & $10(21,7)$ \\
\hline & \multicolumn{3}{|c|}{$\begin{array}{l}\text { Frequência dos impactos sobre as atividades diárias por causa } \\
\text { da ocorrência dos sinais e sintomas de rinite alérgica e/ou asma }\end{array}$} \\
\hline & $\begin{array}{l}\text { Nunca } \\
\text { n (\%) }\end{array}$ & $\begin{array}{l}\text { Às vezes } \\
\text { n }(\%)\end{array}$ & $\begin{array}{c}\text { Frequentemente } \\
\text { n (\%) }\end{array}$ \\
\hline Acorda a noite e/ou perde o sono & $12(26,1)$ & $24(52,5)$ & $10(21,7)$ \\
\hline
\end{tabular}




\begin{tabular}{l|c|c|c}
\hline Ao acordar tem sinais e sintomas & $10(21,7)$ & $31(67,4)$ & $5(10,9)$ \\
\hline Ausência no colégio & $22(47,8)$ & $22(47,8)$ & $2(4,3)$ \\
\hline Dificuldade de concentração & $14(30,4)$ & $22(47,8)$ & $10(21,7)$ \\
\hline Interferência nas atividades de lazer & $11(28,9)$ & $31(67,4)$ & $4(8,7)$ \\
\hline Irritação & $7(15,2)$ & $20(43,5)$ & $19(41,3)$ \\
\hline Uso de medicamentos & $1(2,2)$ & $29(63)$ & $16(34,8)$ \\
\hline Internação no hospital & $30(65,2)$ & $15(32,6)$ & $1(2,2)$ \\
\hline
\end{tabular}

Fonte: Autores (2017).

Os principais impactos dos sinais e sintomas de rinite alérgica e/ou asma nas atividades diárias (Tabela 1) foram irritação $(41,3 \% ; n=19)$ e uso de medicamentos $(34,8 \% ; n=16)$. Cabe ressaltar que apenas uma parcela entre 20 a $30 \%$ dos estudantes relatou que nunca apresentaram alterações no sono e/ou problemas na concentração por causa destas doenças. Grande parte do perfil dos estudantes foi classificada como baixo impacto (47,8\%; $n=22)$. Porém, as doenças mostraram exercer alto impacto em 28,3\% ( $n=13$ ). Também não houve diferença significativa e associação entre os tipos de doenças (rinite alérgica, asma e rinite/asma) e os impactos da sintomatologia no cotidiano ( $p=0,796 ; r=0,094)$.

A análise da associação entre frequência dos sinais e sintomas e impactos nas atividades diárias (Tabela 2) revelou que, dentre os estudantes que foram classificados na faixa de alta frequência de manifestação da sintomatologia, 66,7\% também apresentaram maiores impactos no cotidiano $(p<0,05)$. O padrão se repetiu no cruzamento das faixas baixas. Dessa forma, 0 nível de impacto nas atividades diárias se correlacionou positivamente com o nível de frequência de sinais e sintomas $\left(T_{T} b=0,503\right)$.

Tabela 2. Frequências absoluta e relativa das faixas do impacto sobre atividades diárias em relação às faixas de manifestação dos sinais e sintomas de rinite alérgica e/ou asma dos estudantes do ensino médio regular que utilizam medicamentos para estas doenças ( $n=46$ ), Simão Dias, SE, 2017

\begin{tabular}{|c|c|c|c|c|c|}
\hline \multirow{2}{*}{ Variáveis } & \multicolumn{3}{|c|}{$\begin{array}{c}\text { Faixas do impacto } \\
\text { sobre as atividades diárias }\end{array}$} & \multirow{2}{*}{$\mathbf{p}$} & \multirow{2}{*}{$T^{b}$} \\
\hline & $\begin{array}{l}\text { Baixo } \\
\text { n (\%) }\end{array}$ & $\begin{array}{l}\text { Médio } \\
\text { n (\%) }\end{array}$ & $\begin{array}{l}\text { Alto } \\
\text { n (\%) }\end{array}$ & & \\
\hline $\begin{array}{l}\text { Faixas da manifestação } \\
\text { dos sinais e sintomas }\end{array}$ & & & & $0,003^{*}$ & 0,503 \\
\hline Baixo & $15(71,4)^{a}$ & $6(46,2)^{a}$ & $1(8,3)^{b}$ & & \\
\hline Médio & $4(19)$ & $4(30,8)$ & $3(25)$ & & \\
\hline Alto & $2(9,5)^{a}$ & $3(28,3)^{\mathrm{a}}$ & $8(66,7)^{b}$ & & \\
\hline
\end{tabular}

Fonte: Autores (2017).

Notas: 'As frequências relativas estão representadas em relação aos impactos sobre as atividades diárias/frequência da manifestação de sinais e sintomas.

${ }^{2} \mathrm{p}=$ valor de $\mathrm{p} ; \mathrm{T} \mathrm{b}=$ coeficiente de Kendall'sTau-b.

3*Diferença significativa $p<0,05$.

${ }^{4}$ Diferença significativa entre as proporções das colunas está representada por letras distintas $(a, b<0,05)$. 
Considerando a relação das variáveis com o sexo (Tabela 3), as adolescentes apresentaram prevalências de 51,6\% para rinite alérgica $(n=16)$ e 9,7\% para coexistência rinite alérgica/asma $(n=3)$. A ocorrência de sinais e sintomas com frequência alta foi maior no sexo feminino $(35,5 \% ; n=11)$ quando comparada com a do sexo masculino $(p<0,05)$. Por outro lado, não houve diferença e nem associação com os impactos nas atividades diárias.

Tabela 3. Frequências absoluta e relativa relacionadas às doenças associadas ao uso de medicamentos para rinite alérgica e/ou asma, às faixas da manifestação de sinais e sintomas e dos impactos sobre as atividades diárias em relação ao sexo dos estudantes do ensino médio regular ( $n=46)$, Simão Dias, SE, 2017

\begin{tabular}{|c|c|c|c|c|}
\hline \multirow[b]{2}{*}{ Variáveis } & \multicolumn{2}{|c|}{ Sexo } & \multirow[b]{2}{*}{$\mathbf{p}$} & \multirow[b]{2}{*}{$\mathbf{r}$} \\
\hline & $\begin{array}{c}\text { Feminino } \\
n(\%)\end{array}$ & $\begin{array}{c}\text { Masculino } \\
\text { n (\%) }\end{array}$ & & \\
\hline Doença associada ao uso de medicamento & & & 0,178 & 0,060 \\
\hline Rinite alérgica & $16(51,6)$ & $7(46,7)$ & & \\
\hline Asma & $12(38,7)$ & $6(40)$ & & \\
\hline Rinite alérgica e asma & $3(9,7)$ & $2(13,3)$ & & \\
\hline \multicolumn{3}{|c|}{ Faixas da manifestação dos sinais e sintomas } & 0,101 & $-0,229$ \\
\hline Baixo & $13(41,9)$ & $8(53,3)$ & & \\
\hline Médio & $7(22,6)$ & $6(46,2)$ & & \\
\hline Alto & $11(35,5)^{\mathrm{a}}$ & $1(6,7)^{b}$ & & \\
\hline \multicolumn{2}{|c|}{ Faixas do impacto sobre as atividades diárias } & & 0,504 & $-0,167$ \\
\hline Baixo & $13(41,9)$ & $9(60)$ & & \\
\hline Médio & $8(25,8)$ & $3(20)$ & & \\
\hline Alto & $10(32,3)$ & $3(20)$ & & \\
\hline
\end{tabular}

Fonte: Autores (2017)

Notas: ${ }^{1}$ As frequências relativas estão representadas em relação às variáveis da linha/sexo.

${ }^{2} \mathrm{p}=$ valor de $\mathrm{p}$; $r=$ coeficiente de Pearson.

${ }^{3}$ Diferença significativa entre as proporções das colunas está representada por letras distintas $(a, b<0,05)$.

No que se refere ao perfil do uso de medicamentos para rinite alérgica, as vias de administração mais comuns foram oral $(n=9 ; 32,1 \%)$, nasal $(n=8 ; 17,4 \%)$ e combinação oral/nasal $(n=8 ; 17,4 \%)$. Todos os medicamentos utilizados eram produzidos industrialmente. Não foi relatado nenhum manipulado em farmácia magistral e/ou homeopática, bem como o uso de chás para o controle da doença. Quando foram questionados quanto ao nome dos medicamentos que utilizavam apenas 14,3\% não conseguiram dizer e não os reconheceram por meio de fotos $(n=4)$. Cabe ressaltar que $46,4 \%$ afirmaram usar o medicamento para prevenir as crises alérgicas $(n=13)$.

Dentre aqueles que possuíam rinite alérgica $(n=28), 53,6 \%$ afirmaram fazer o uso de anti-histamínicos $(n=15)$. Os medicamentos com corticosteroides foram relatados por $42,9 \%(n=12)$. Com relação à utilização de descongestionantes nasais, $39,3 \%$ confirmaram o uso dos mesmos $(n=11)$. Uma proporção pequena $(7,1 \% ; n=2)$ relatou o uso de colírios com corticosteroides. Quanto à distribuição da frequência de associação das diferentes classes de medicamentos, 46,4\% utilizam apenas um tipo ( $n=13)$ e 39,3\% associam mais ( $n=11)$. A proporção de estudantes que usam corticosteroides foi maior dentre 
aqueles que se situavam na faixa de frequência alta de sinais e sintomas $(81,8 \% ; n=9 ; p<0,05)$. Por outro lado, o grau de impacto nas atividades diárias não se relacionou com a classe de medicamento utilizada (Tabela 4).

Tabela 4. Frequências absoluta e relativa relacionadas às faixas da manifestação dos sinais e sintomas de rinite alérgica e do impacto sobre as atividades diárias em relação ao perfil do uso de medicamentos por estudantes do ensino médio regular ( $n=46$ ), Simão Dias, SE, 2017

\begin{tabular}{|c|c|c|c|}
\hline \multirow[b]{2}{*}{ Variáveis } & \multicolumn{3}{|c|}{ Faixas da manifestação dos sinais e sintomas de rinite alérgica } \\
\hline & $\begin{array}{l}\text { Baixo } \\
\text { n (\%) }\end{array}$ & $\begin{array}{c}\text { Médio } \\
\text { n (\%) }\end{array}$ & $\begin{array}{c}\text { Alto } \\
\text { n (\%) }\end{array}$ \\
\hline \multicolumn{4}{|l|}{ Anti-histamínico } \\
\hline Usa & $5(45,5)$ & $3(50)$ & $7(63,6)$ \\
\hline Não usa & $6(54,5)$ & $3(50)$ & $4(36,4)$ \\
\hline \multicolumn{4}{|l|}{ Corticosteroide } \\
\hline Usa & $1(9,1)^{\mathrm{a}}$ & $2(33,3)$ & $9(81,8)^{a}$ \\
\hline Não usa & $10(90,9)^{b}$ & $4(66,7)$ & $2(18,2)^{b}$ \\
\hline \multicolumn{4}{|l|}{ Descongestionantes nasais } \\
\hline Usa & $2(18,2)$ & $4(66,7)$ & $5(45,5)$ \\
\hline Não usa & $9(81,8)$ & $2(36,4)$ & $6(54,5)$ \\
\hline \multicolumn{4}{|l|}{ Associação } \\
\hline Apenas uma classe & $6(46,2)^{a}$ & $1(12,5)$ & - \\
\hline Duas classes & $3(23,1)$ & $2(25)$ & $1(33,3)$ \\
\hline \multirow[t]{3}{*}{ Três classes } & $4(30,8)$ & $5(62,5)$ & $2(66,7)$ \\
\hline & \multicolumn{3}{|c|}{ Faixas do impacto da rinite alérgicas sobre as atividades diárias } \\
\hline & $\begin{array}{l}\text { Baixo } \\
\text { n (\%) }\end{array}$ & $\begin{array}{l}\text { Médio } \\
\text { n (\%) }\end{array}$ & $\begin{array}{l}\text { Alto } \\
\text { n (\%) }\end{array}$ \\
\hline \multicolumn{4}{|l|}{ Anti-histamínico } \\
\hline Usa & $6(46,2)$ & $5(71,4)$ & $4(50)$ \\
\hline Não usa & $7(53,8)$ & $2(28,6)$ & $4(50)$ \\
\hline \multicolumn{4}{|l|}{ Corticosteroide } \\
\hline Usa & $4(30,8)$ & $3(42,9)$ & $5(62,5)$ \\
\hline Não usa & $9(69,2)$ & $4(57,1)$ & $3(37,5)$ \\
\hline \multicolumn{4}{|l|}{ Descongestionantes nasais } \\
\hline Usa & $5(38,5)$ & $1(14,3)$ & $5(62,5)$ \\
\hline Não usa & $8(61,5)$ & $6(85,7)$ & $3(37,5)$ \\
\hline \multicolumn{4}{|l|}{ Associação } \\
\hline Apenas uma classe & $6(46,2)$ & $3(42,9)$ & $4(50)$ \\
\hline Duas classes & $2(15,4)$ & $3(42,9)$ & $3(37,5)$ \\
\hline Três classes & $2(15,4)$ & - & $1(12,5)$ \\
\hline
\end{tabular}

Fonte: Autores (2017).

Nota: 'As frequências relativas estão representadas em relação às faixas da manifestação dos sinais e sintomas da rinite alérgica/variáveis das linhas. 
Sobre a crise asmática, 30,4\% ( $n=7)$ afirmaram ter crises todos os meses do ano e 26,1\% ( $n=6)$ entre 6 e 11 meses por ano. Em relação à influência da época do ano para a manifestação da crise, 78,3\% ( $n=18)$ relataram ter uma época mais comum. É importante ressaltar que 39,1\% não souberam dizer o nome do medicamento para crise e nem o reconheceu (n=9). Além disso, a maioria não porta o medicamento para as crises $(52,2 \% ; n=12)$. Os inaladores e/ou aerossóis são utilizados durante a crise por $52,2 \%(n=12)$, os demais não sabem ou não usam. Não houve diferença entre as faixas de impactos nas atividades diárias em relação ao uso dos medicamentos para crise asmática (Tabela 5).

Com relação ao nome dos medicamentos para manutenção, a maioria dos estudantes não sabia, mas alguns reconheceram (30\%; $n=7)$. Foi encontrada maior proporção de uso dentre aqueles que possuíam frequência elevada de sinais e sintomas (100\%; $n=3 ; p<0,05)$. Por outro lado, não houve diferença entre as faixas de impactos nas atividades diárias em relação ao uso dos medicamentos de manutenção (Tabela 5).

Tabela 5. Frequências absoluta e relativa relacionadas às faixas da manifestação dos sinais e sintomas de asma e do impacto sobre as atividades diárias em relação ao perfil do uso de medicamentos por estudantes do ensino médio regular ( $n=46)$, Simão Dias, SE, 2017

\begin{tabular}{|c|c|c|c|}
\hline \multirow[b]{2}{*}{ Variáveis } & \multicolumn{3}{|c|}{ Faixas da manifestação dos sinais e sintomas de asma } \\
\hline & $\begin{array}{l}\text { Baixo } \\
\text { n (\%) }\end{array}$ & $\begin{array}{l}\text { Médio } \\
\text { n (\%) }\end{array}$ & $\begin{array}{l}\text { Alto } \\
\text { n (\%) }\end{array}$ \\
\hline \multicolumn{4}{|c|}{ Medicamento para crise asmática } \\
\hline Usa & $4(40)$ & $6(60)$ & $2(66,7)$ \\
\hline Não usa ou não sabe & $6(60)$ & $4(40)$ & $1(33,3)$ \\
\hline \multicolumn{4}{|l|}{ Medicamento para manutenção } \\
\hline Usa & $1(10)^{\mathrm{a}}$ & $3(30)^{\mathrm{a}}$ & $3(100)^{b}$ \\
\hline \multirow[t]{3}{*}{ Não usa ou não sabe } & $9(90)^{a}$ & $7(70)^{a}$ & $-b$ \\
\hline & \multicolumn{3}{|c|}{$\begin{array}{l}\text { Faixas do impacto dos sinais e sintomas de asma sobre as } \\
\text { atividades diárias }\end{array}$} \\
\hline & $\begin{array}{l}\text { Baixo } \\
\text { n (\%) }\end{array}$ & $\begin{array}{l}\text { Médio } \\
\text { n (\%) }\end{array}$ & $\begin{array}{l}\text { Alto } \\
\text { n (\%) }\end{array}$ \\
\hline \multicolumn{4}{|c|}{ Medicamento para crise asmática } \\
\hline Usa & - & - & $2(25)$ \\
\hline Não usa ou não sabe & $11(100)$ & $4(100)$ & $6(75)$ \\
\hline \multicolumn{4}{|l|}{ Medicamento para manutenção } \\
\hline Usa & $2(18,2)$ & $1(25)$ & $4(50)$ \\
\hline Não usa ou não sabe & $9(81,8)$ & $3(75)$ & $4(50)$ \\
\hline
\end{tabular}

Fonte: Autores (2017).

Nota: ${ }^{1}$ As frequências relativas estão representadas em relação às faixas da manifestação dos sinais e sintomas da rinite alérgica/variáveis das linhas. 


\section{DISCUSSÃo}

Rinite alérgica e asma são doenças inflamatórias crônicas das vias aéreas que afetam grande parte da população mundial. Neste estudo, a maioria dos adolescentes portadores destas doenças relatou usar medicamentos para prevenção e/ ou controle das mesmas. É importante destacar que não foi avaliada a questão da automedicação e/ou prescrição médica. O resultado está de acordo com a faixa de proporção da população com acesso a fármacos essenciais para asma e/ou rinite alérgica apresentada em outras pesquisas realizadas no Brasil (50-80\%) e em outros países que, por sua vez, mostram variações consideráveis $(<50 \mathrm{a}>95 \%)^{(8,9)}$. Estas diferenças estão relacionadas às diferentes abordagens metodológicas como, principalmente, ao uso de medicamentos considerando automedicação e/ou prescrição médica e de instrumentos de pesquisa diferentes, ao perfil socioeconômico e organização dos serviços de saúde, como existência de políticas de saúde específicas para estas doenças. ${ }^{(10,11)}$

Dentre os adolescentes que utilizam medicamentos para estas doenças, as prevalências de rinite alérgica e asma foram de $50 \%$ e $41,8 \%$, respectivamente, corroborando com o fato de que a rinite alérgica pode ser considerada a enfermidade de maior frequência entre as doenças respiratórias crônicas ${ }^{(6,12)}$. Considerando ainda que a rinite alérgica apresenta estreita relação com a asma ${ }^{(6,12,14)}$, também observou-se, neste estudo, a coexistência em $\approx 30 \%$ dos casos. São demonstradas cada vez mais evidências consistentes que estas doenças não possuem apenas bases epidemiológicas comuns, mas também genéticas, histopatológicas, imunológicas e clínicas( ${ }^{(6)}$. Os principais impactos desta associação se referem à maior severidade da asma e ao aumento dos gastos com o tratamento. ${ }^{(6,14)}$

A frequência das manifestações de sinais e sintomas foi diferente entre os sexos, uma vez que as adolescentes a apresentaram mais elevada. Outros estudos também demonstraram maior prevalência de sintomas, incluindo aqueles de maior gravidade, em adolescentes do sexo feminino. Como fatores interferentes sugeriram-se os hormonais e, na discussão de gênero, o maior contato com serviços de saúde e abertura das adolescentes no que se refere aos problemas de saúde ${ }^{(15)}$. Além disso, foi discutido o reflexo das questões culturais em função da maior exposição aos fatores ambientais intradomiciliares devido à responsabilidade com as tarefas domésticas. ${ }^{(16)}$

É importante ressaltar que, neste estudo, houve relação entre a alta frequência dos sinais/sintomas e maior impacto nas atividades diárias. Outra pesquisa evidenciou que $24,3 \%$ dos portadores de rinite e/ou asma referiram ser muito afetados pela doença ${ }^{(17)}$, ou seja, uma proporção semelhante à deste trabalho (26\%). De modo geral, os pacientes sentem-se incomodados pelos sintomas propriamente ditos, particularmente pela obstrução nasal, coriza e espirros. Ficam aborrecidos por não conseguirem dormir bem à noite, apresentam limitações nas atividades diárias e sentem-se frustrados e irritados. Os adolescentes vivenciam problemas de modo semelhante aos adultos, que afetam, principalmente, a rotina dos estudos. ${ }^{(18)}$

Com relação à rinite alérgica, a maioria dos estudantes foi classificada no perfil baixo de sinais e sintomas e também de baixo impacto nas atividades diárias. De acordo com a intensidade das manifestações clínicas e dos impactos sobre a qualidade de vida, a rinite alérgica é classificada como intermitente ou persistente e ainda como leve ou moderada/grave ${ }^{(19)}$. Esta caracterização é fundamental porque reflete no planejamento da conduta terapêutica. ${ }^{(2)}$

Considerando a classificação da rinite alérgica, foram identificados problemas na adesão ao tratamento farmacológico e, em alguns casos, uso inadequado dos medicamentos. Os medicamentos mais utilizados na rinite alérgica são os antihistamínicos orais e os corticosteroides nasais ${ }^{(2)}$. Alguns adolescentes possuíam alta frequência de sinais e sintomas, bem como apresentavam elevado impacto nas atividades diárias e não faziam uso de corticosteroides.

Assim como ocorre nos casos de rinite alérgica, o tratamento da asma deve ser baseado na classificação da gravidade e no estado de controle da doença( ${ }^{(2)}$. Segundo o presente estudo, uma atenção especial deve ser dada àqueles adolescentes 
que possuem de média a alta frequência de sinais e sintomas da asma, uma vez que a maioria não conhece ou não reconhece a medicação utilizada para manutenção e, alguns, não portam o medicamento para crise. Além disso, foram identificados estudantes que relatam o uso de medicamentos para o controle da asma, porém ainda possuem média e alta frequência de sinais e sintomas, o que pode estar relacionado ao tratamento incorreto.

O tratamento atual da asma é direcionado para controlar os sintomas e prevenir exacerbações. Entretanto, na maioria das vezes os pacientes não seguem o tratamento adequadamente ou, simplesmente, abandonam a terapia por conta própria ${ }^{(7)}$ e, consequentemente, acabam manifestando várias crises durante o ano, como foi relatado por alguns estudantes nesta pesquisa. O déficit de conhecimento sobre as doenças pode levar à falsa sensação de cura que se relaciona com a redução dos sinais e sintomas em resposta ao tratamento farmacológico ${ }^{(20)}$. No caso especial da asma, o nível de conhecimento interfere diretamente no manejo adequado e no número de ingressos nos serviços de saúde ${ }^{(20)}$. Portanto, as diferenças entre tratamento broncodilatador sintomático e tratamento de manutenção regular devem ser enfatizadas ${ }^{(4)}$.

Para asma, a via de escolha para o tratamento é a inalatória, cuja principal vantagem está relacionada ao alcanço seletivo dos pulmões, o que resulta na elevação da concentração do fármaco nas vias aéreas e diminui os efeitos adversos sistêmicos. Entretanto, a dificuldade na técnica de utilização dos dispositivos inalatórios pode levar a uma redução da eficácia dos medicamentos e se tornar um empecilho na adesão do paciente ao tratamento ${ }^{(4)}$. Estudos demonstraram que a maior parte dos indivíduos portadores da doença não sabiam utilizar corretamente as medicações, em especial a "bombinha". ${ }^{20,21)}$

Cabe ressaltar que o tratamento farmacológico não reduz a necessidade de ações educativas para diminuir a exposição aos fatores desencadeantes e para o controle da doença. Dessa forma, todos os pacientes com rinite alérgica e/ou asma, bem como os familiares devem receber orientações sobre a doença.

De acordo com este trabalho, o que mais conta para a utilização dos medicamentos é a manifestação dos sinais e sintomas e não o impacto sobre a rotina diária. Por se tratarem de doenças que não têm cura, é preciso que haja um tratamento adequado e com o devido acompanhamento do profissional de saúde. Quando o paciente possui conhecimento quanto ao manejo, controle e cuidados com a doença há uma melhora na prevenção das crises, aumentando cada vez mais a qualidade de vida. ${ }^{(22)}$

\section{CONCLUSÃO}

A taxa de utilização de medicamentos para rinite alérgica e/ou asma foi elevada quando comparada com a realidade de outros estudos. No entanto, foram identificados alguns problemas na adequação do tratamento farmacológico e uso correto das medicações para estas doenças que, por sua vez, resultaram na alta frequência da ocorrência de sinais e sintomas e alto impacto no cotidiano de alguns adolescentes.

As manifestações clínicas foram classificadas, em sua maioria, na faixa de baixa frequência de sinais e sintomas e baixo impacto nas atividades diárias, o que pode indicar o próprio perfil sintomatológico da doença ou controle e manejo adequados. Uma atenção especial deve ser voltada às adolescentes do sexo feminino, uma vez que apresentaram maior frequência de exacerbações agudas.

Destaca-se, portanto, a importância da implementação de estratégias de orientação voltadas para rinite alérgica e asma, bem como a sensibilização dos adolescentes quanto ao autocuidado a fim de melhorar o controle das doenças e reduzir os impactos das mesmas sobre a qualidade de vida. Cabe ressaltar também a importância dessas ações para minimizar/eliminar os riscos provenientes do uso incorreto desses medicamentos. 


\section{REFERÊNCIAS}

1. Silva ECF. Rinite alérgica e comorbidades. Rev. HUPE [Internet]. 2008 [cited 2016 Aug. 15];7(2):12-23. Available from: $<$ http://revista.hupe.uerj.br/detalhe_artigo.asp?id=200>

2. Ministério da Saúde. Cadernos de Atenção Básica - Doenças respiratórias crônicas [Internet]. Brasília: Ministério da Saúde; 2010 [cited 2016 Aug 2]. Available from: <http://bvsms.saude.gov.br/bvs/publicacoes/doencas_respiratorias_cronicas.pdf>

3. Silva ECF, Dias GAC. Patogenia da asma. Rev. HUPE [Internet]. 2013 [cited 2016 Aug. 15];12(2):31-40. Available from: $<$ http://revista.hupe.uerj.br/detalhe_artigo.asp?id=391>

4. Simões LZ. Atenção farmacêutica ao paciente portador de Asma e Doença Pulmonar Obstrutiva Crônica: coletânea de estudos sobre adesão, uso de inaladores, sistematização da atenção e perfil farmacoepidemiológico [dissertation]. São Paulo: Universidade de São Paulo; 2015 [cited 2016 Aug. 2]. 128 p. Available from: <http://www.teses.usp.br/teses/ disponiveis/5/5160/tde-03022016-091334/pt-br.php>

5. Rizzo LA, Cruz AA. Asma e rinite, uma mesma doença? Rev. Bras. Alerg. Imunopatol. [internet]. 2007 [cited 2016 Sep. 3]; 30 (2):41-6. Available from: <http://www.asbai.org.br/revistas/Vol302/asma_e_rinite.pdf>

6. ASBAI, ABORL-CCF. III Consenso Brasileiro sobre Rinites. Braz. J. Otorhinolaryngol. [Internet]. 2012 [cited 2017 Feb 1];1-51. Available from: <http://www.aborlccf.org.br/consensos/Consenso_sobre_Rinite-SP-2014-08.pdf>.

7. Diretrizes da Sociedade Brasileira de Pneumologia e Tisiologia para o Manejo da Asma. J. Bras. Pneumol. [Internet]. 2012 [cited 2016 Aug. 20];38:1-46. Available from: <http://www.jornaldepneumologia.com.br/pdf/suple_200_70_38_completo_ versao_corrigida_04-09-12.pdf>

8. The International Study of Asthma and Allergies in Childhood (ISAAC) Steering Committee. Worldwide variation in prevalence of symptoms of asthma, allergic rhinoconjunctivitis, and atopic eczema: ISAAC. Lancet [Internet]. 1998 [cited 2017 Feb. 1]; 351:1225-32. Available from: <https://www.ncbi.nlm.nih.gov/pubmed/9643741https://www.ncbi.nlm.nih. gov/pubmed/9643741>

9. Solé D, Wandalsen GF, Camelo-Nunes IC, Naspitz CK; ISAAC Brazilian Group. Prevalence of symptoms of asthma, rhinitis, and atopic eczema among Brazilian children and adolescents identified by the International Study of Asthma and Allergies in Childhood (ISAAC) - Phase 3. J Pediatr [Internet]. 2006 [cited 2017 Feb 1]; 82:341-6. Available from: <www.scielo.br/ scielo.php?script=sci_arttext\&pid=S0021-75572006000600006>

10. Strachan DP. Family size, infection and atopy: the first decade of the "hygiene hypothesis". Thorax [Internet]. 2000 [cited 2017 Feb. 1]; 55:2-10. Available from: <https://www.ncbi.nlm.nih.gov/pmc/articles/PMC1765943/>

11. von Mutius E. The environmental predictors of allergic disease. J Allergy Clin. Immunol. [Internet]. 2000 [cited 2017 Apr. 1]; 105:9-19. Available from:<https://www.ncbi.nlm.nih.gov/pubmed/10629447>

12. Luna MFG, Fischer GB, Luna JRG, Silva MGC, Almeida PC, Chiesa D. Comparação temporal das prevalências de asma e rinite em adolescentes em Fortaleza, Brasil. J. Bras. Pneumol [Internet]. 2013 [cited 2017 Apr. 2]; 39(2):128-137. Available from: <http://www.scielo.br/pdf/jbpneu/v39n2/v39n2a03.pdf>

13. Luna MFG, Almeida PC, Silva MGC. Prevalência e associação de asma e rinite em adolescentes de 13 a 14 anos de Fortaleza, Ceará, Brasil. Cad. Saúde Pública [Internet]. 2011 [cited 2017 Apr. 2]; 21(7):103-112. Available from: <http://www.scielo.br/ scielo.php?script=sci_arttext\&pid=S0102-311X2011000100011 > 
14. Andrade CR, Camargos PAM, Ibiapina CC, Alvim CG, Drummond A, Vilaça D et al. Comorbidade asma e rinite alérgica: inter-relações entre as vias aéreas superiores e inferiores. Rev. Med. Minas Gerais [Internet]. 2009 [cited 2017 Apr. 10]; 19(4 Supl. 5):S19-S24. Available from: <http://rmmg.org/exportar-pdf/1121/v19n4s5a04.pdf>

15. Medeiros ML, Solé D, Costa ADPV, Andrade ANVF, Mello PKS, Santos DAM et al. Prevalência de asma e rinite entre adolescentes de 13-14 anos em uma capital do Nordeste, de acordo com o questionário do InternationalStudyofAsthmaandAllergies in Childhood (ISAAC). Braz. J. Allergy and Immunol [Internet]. 2014 [cited 2017 March 20]; 2(3):112-8. Available from: <www. bjai.org.br/audiencia_pdf.asp?aid2=694\&nomeArquivo=v2n3a05.pdf>

16. Toledo MF. Prevalência da asma, rinite e eczema em adolescentes de 13 a 14 anos na cidade de Taubaté- SP, através do questionário ISAAC e avaliação de alguns fatores de risco [dissertação]. São Paulo: Universidade de São Paulo/USP. 2007 [cited 2017 March 30]; 70 p. Available from: <http://www.fcmsantacasasp.edu.br/images/Arquivos_medicos/2011/56_1/03_ ao16.pdf>

17. Corti ACR, Banca RO, Miyasaki P, Solé D. Impacto sobre a qualidade de vida e o nível de satisfação com o tratamento da rinite alérgica por crianças e adolescentes acompanhados em serviço de referência. Rev. Bras. Alergia Imunopatol. [Internet]. 2001 [cited 2017 Apr. 3]; 34(5):203-208. Available from: http://www.asbai.org.br/revistas/vol345/N34N5-ao-01.pdf

18. Nunes ICC, SOLÉ D. Rinite alérgica: indicadores de qualidade de vida. J. Bras. Pneumol. [Internet]. 2010 [cited 2017 Apr. 29]; 36(1):124-133. Available from: http://www.scielo.br/scielo.php?script=sci_arttext\&pid=\$1806-37132010000100017

19. Bousquet J, Khaltaev N, Cruz AA, Denburg J, Fokkens WJ, Togias A et al. Allergic Rhinits and its impact on Asthma (ARIA) 2008 update (in collaboration with the World Health Organization, GA(2)LEN and AllerGen). Allergy [Internet]. 2008 [cited 2017 Apr. 3]; 63(Suppl. 86):8-160. Available from: https://www.ncbi.nlm.nih.gov/pubmed/18331513.

20. Betterncourt ARC, Oliveira MA, Fernandes ALG. Educação de pacientes portadores de asma brônquica: atuação da enfermeira. J. Pneumol. 2002; 28(4): 193-200.

21. Vieira JWC, Silva AA, Oliveira FM. Conhecimento e impacto sobre o manejo das crises de pacientes portadores de asma. Rev. Bras. Enf. [Internet]. 2008 [cited 2017 Apr. 20]; 61(6):853-7. Available from: http://www.scielo.br/pdf/reben/v61n6/ a10v61n6.pdf.

22. Angnes MR, Macagnan JBA, Cauduro JM, Silveira M. Asma: uma revisão da literatura. Rev. Saúde Públ. Santa Cat. [Internet]. 2012 [cited 2017 Apr. 3]; 5(3):81-94. Available from: http://esp.saude.sc.gov.br/sistemas/revista/index.php/inicio/article/ viewArticle/146.

Submetido em: 26/04/2017

Aceito em: 30/08/2017

Publicado em: 31/12/2017 